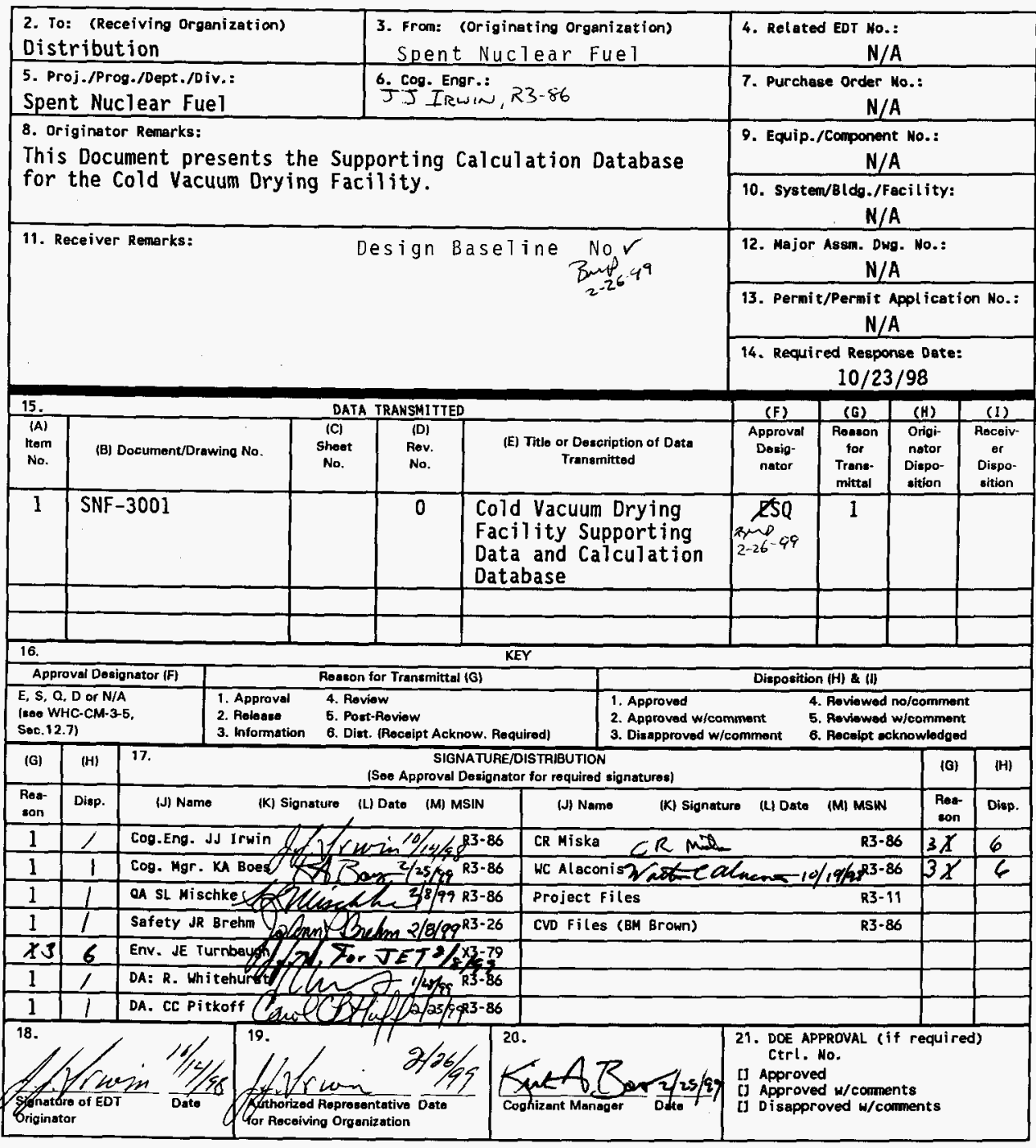

BO-7400-172-2(04/94) GEF097 


\section{SPENT NUCLEAR FUEL PROJECT COLD VACUUM DRYING FACILITY SUPPORTING DATA AND CALCULATION DATABASE}

\section{J. J. Irwin, C. R. Miska}

Numatec Hanford Corporation, Richland, Washington 99352

C. C. Pitkoff, R. Whitehurst

DE\&S Hanford, Inc., Richland, Washington 99352

U.S. Department of Energy Contract DE-AC06-96RL13200

EDT/ECN: 625782

Org Code: 83300

B\&R Code: 39EW70400
UC: 721

Charge Code: 105560

Total Pages: 1315

Key Words: Cold Vacuum Drying, Supporting Calculations Database

Abstract: This document provides a database of supporting calculations for the Cold Vacuum Drying Facility (CVDF). The database was develped in conjunction with HNF-SD-SNF-SAR-002, Safety Analysis Report for the Cold Vacuum Drying Facility, Phase 2, Supporting Installation of Processing Systems (Garvin 1998), The HNF-SD-SNF-DRD-002, 1997, Cold Vacuum Drying Facility Design Requirements, Rev. 2, and the CVDF Summary Design Report. The database contains calculation report entries for a11 process, safety and facility systems in the CVDF, a general CVD operations sequence and the CVDF System Design Descriptions (SDDs). This database been developed for the SNFP CVDF Engineering Organization and shall be updated, expanded, and revised in accordance with future design, construction and startup phases of the CVDF until the CVDF final ORR is approved.

TRADEMARK DISCLAIMER. Reference herein to any specific commercial product, process, or service by trade name, trademark, manufacturer, or otherwise, does not necessarily constitute or imply its endorsement, recommendation, or favoring by the United states Government or any agency thereof or its contractors or subcontractors.

Printed in the United States of America. To obtain copies of this document, contact: Document Control Services, P.0. Box 950, Mailstop H6-08, Richland WA 99352, Phone (509) 372-2420; Fax (509) 376-4989.

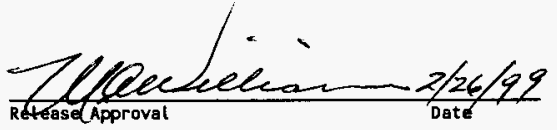

Approved for Public Release 


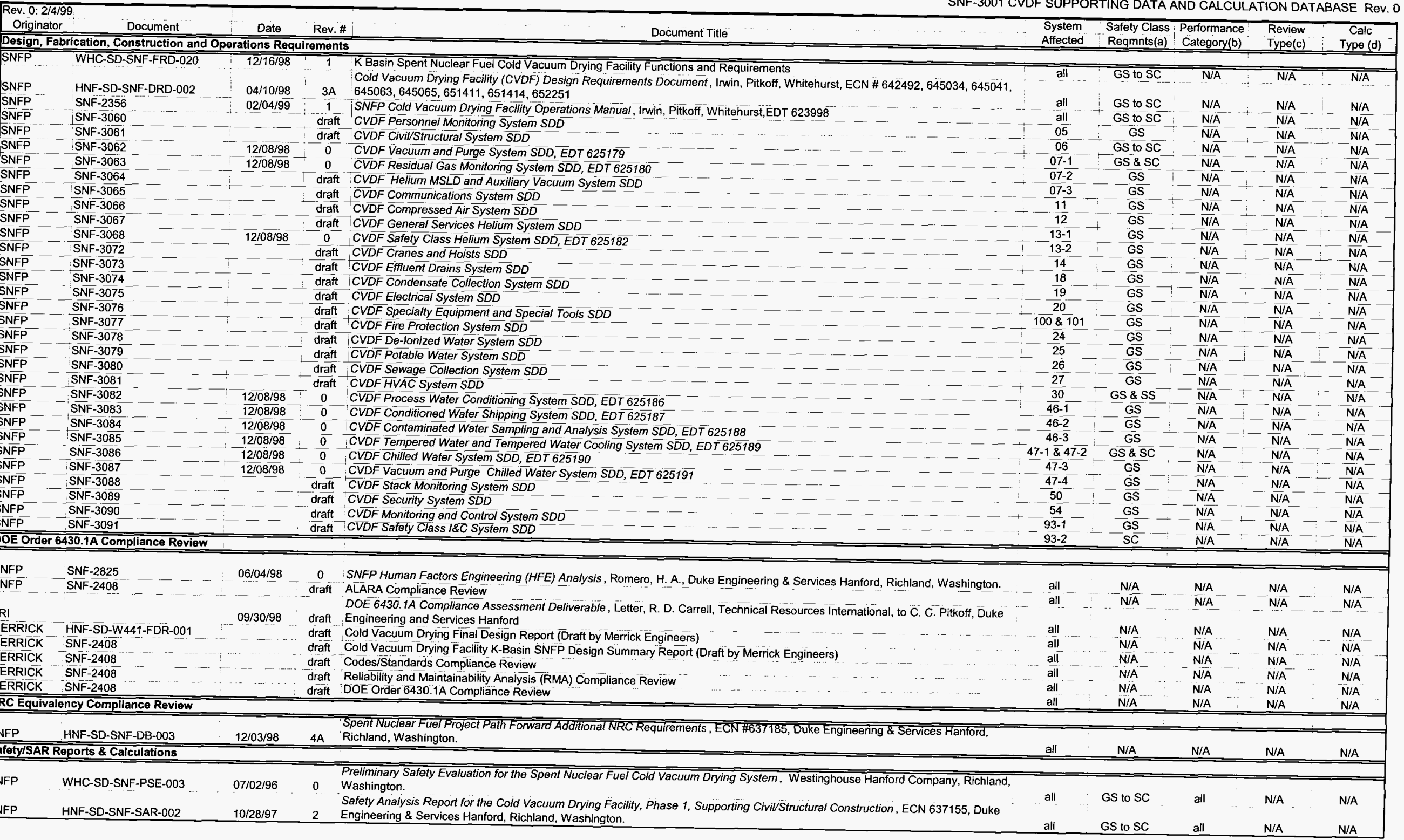




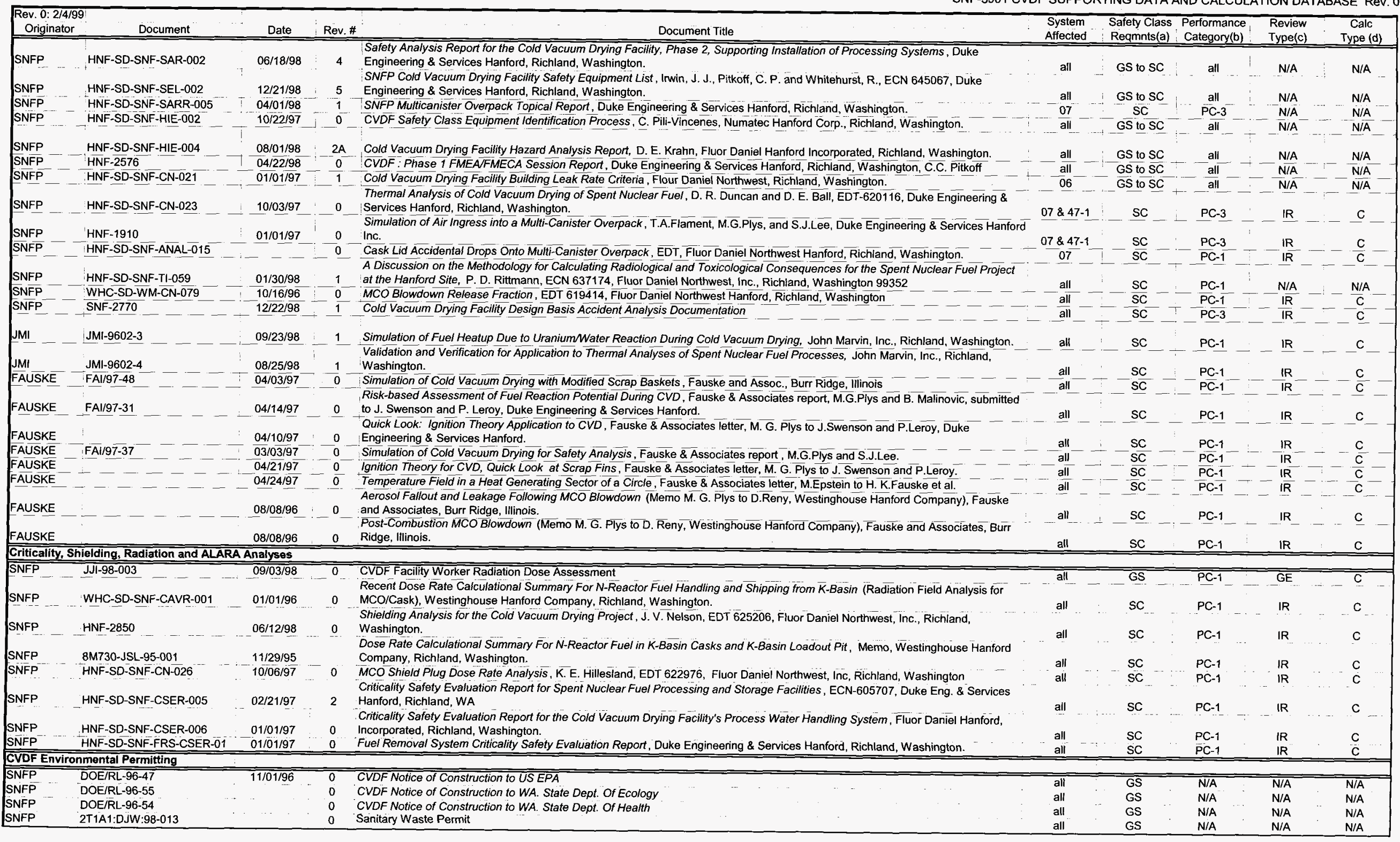




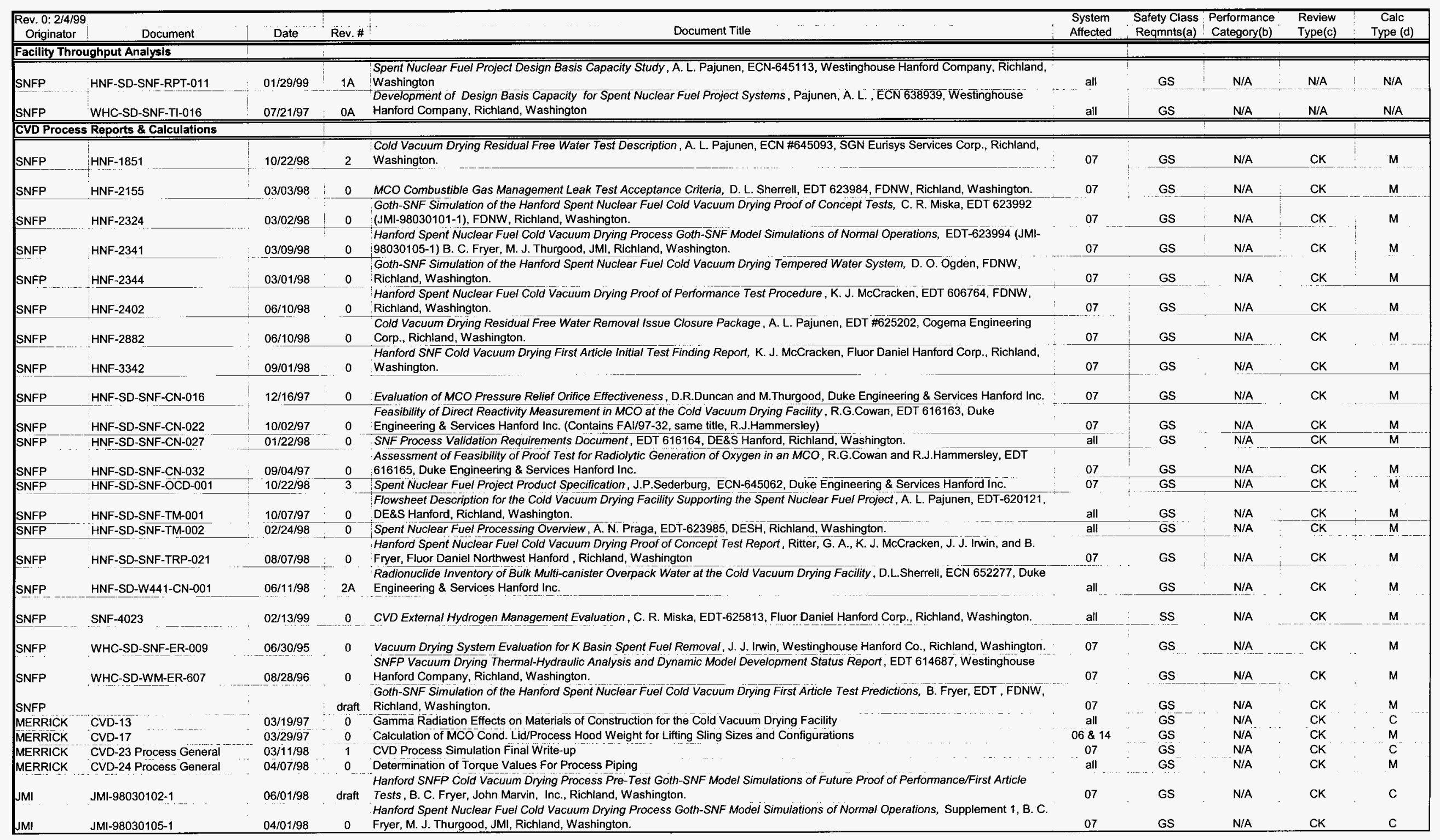


SNF-3001 CVDF SUPPORTING DATA AND CALCULATION DATABASE REV. 0

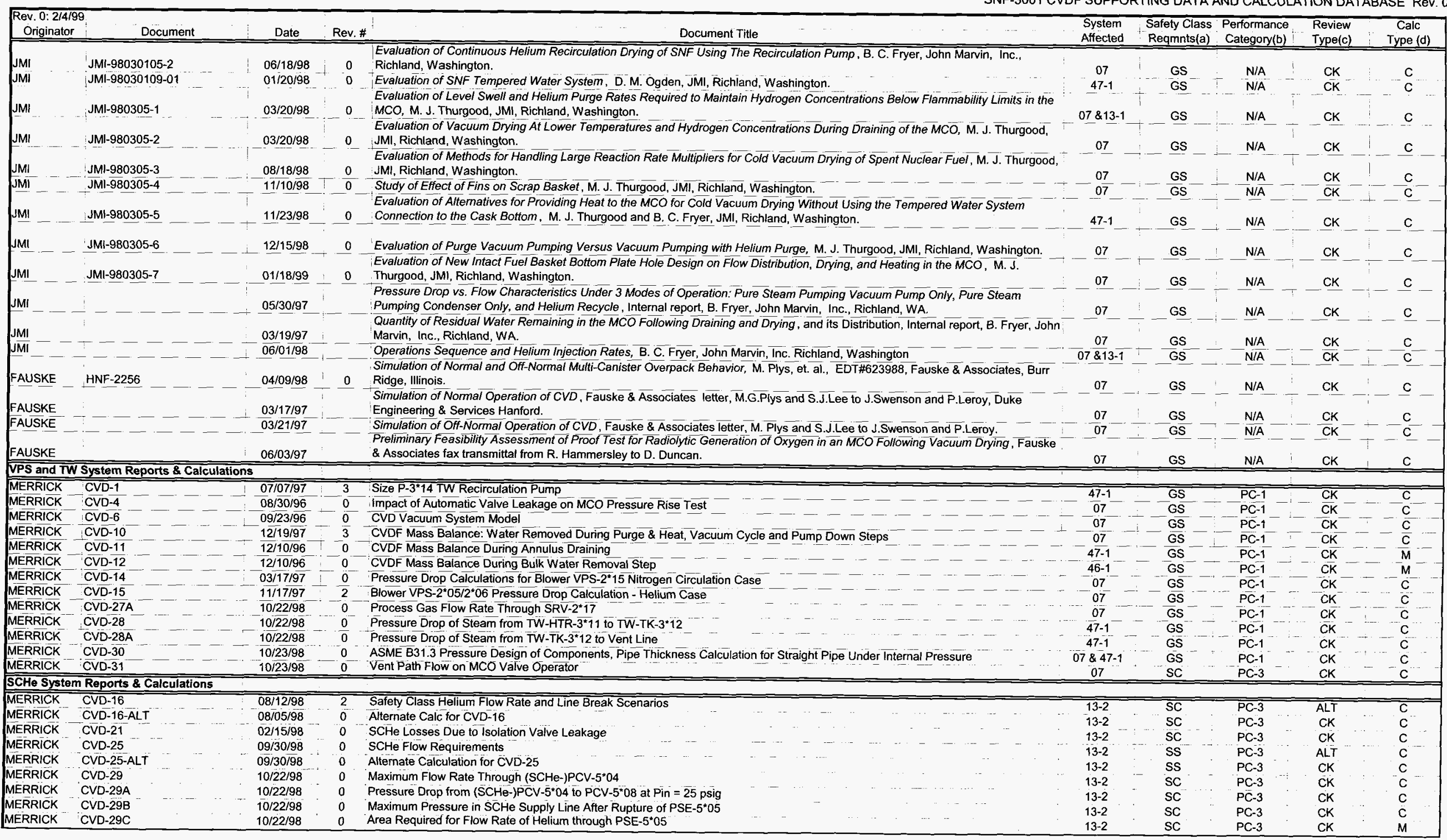


SNF-3001 CVDF SUPPORTING DATA AND CALCULATION DATABASE Rev. 0

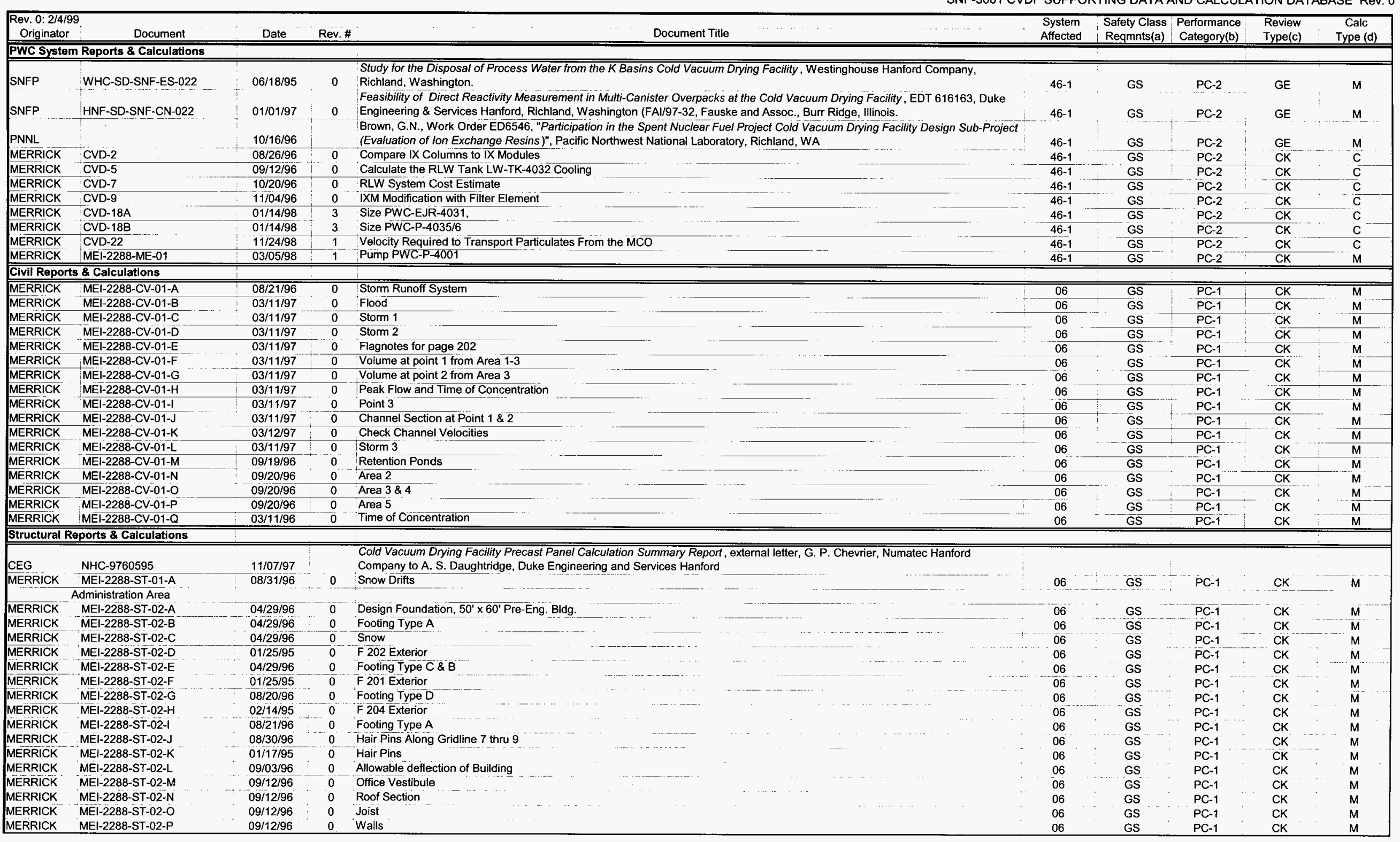


SNF-3001 CVDF SUPPORTING DATA AND CALCULATION DATABASE Rev. 0

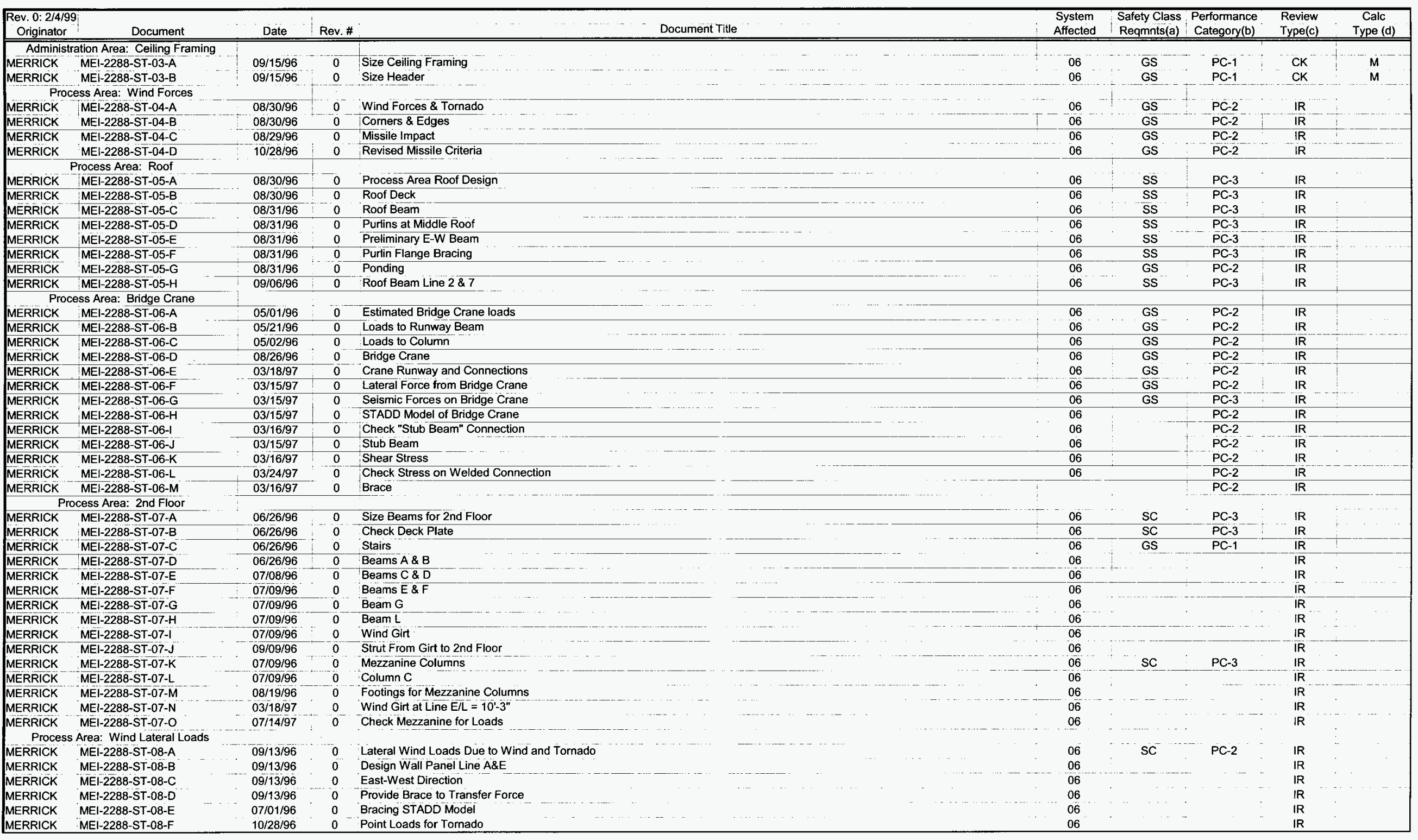




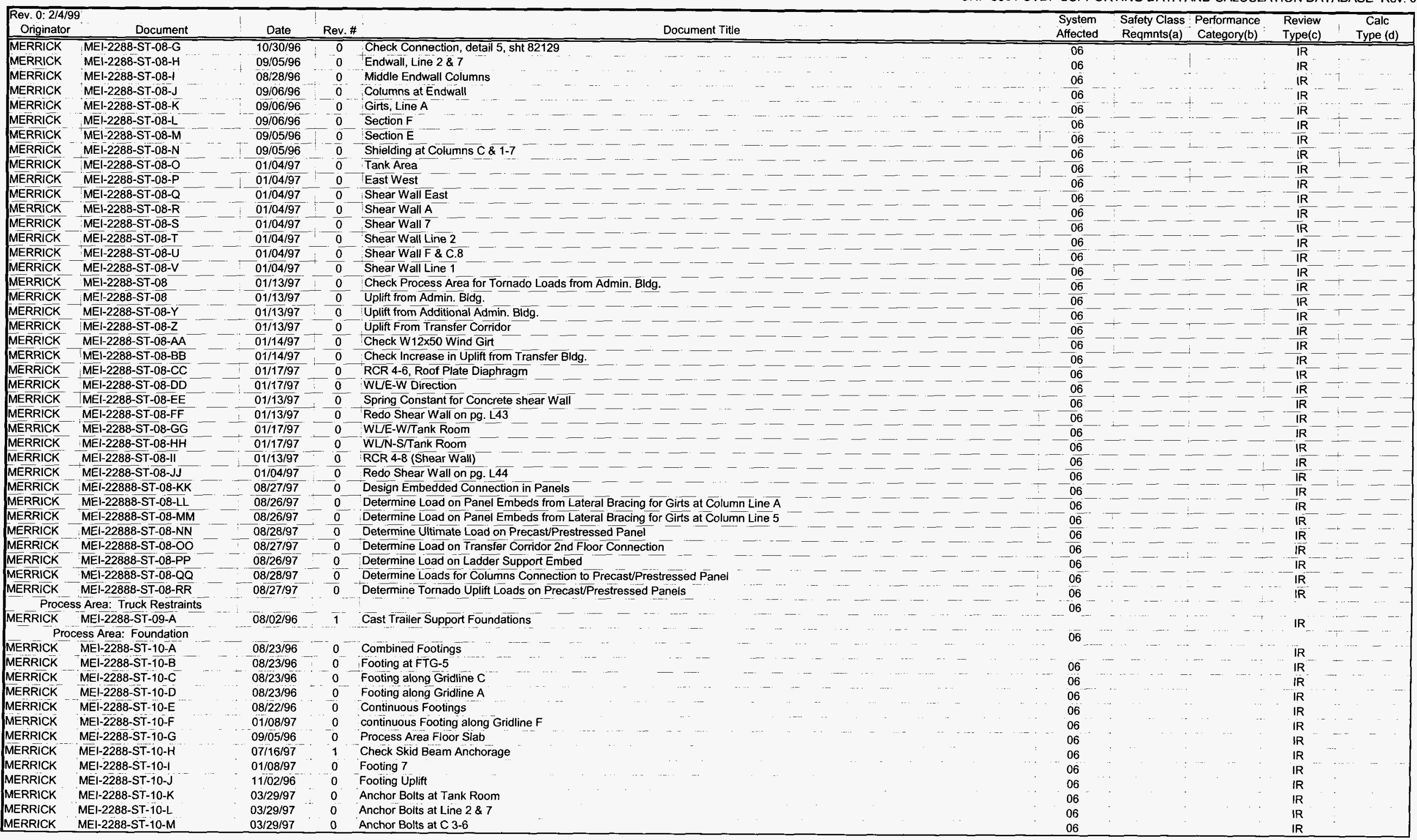


SNF-3001 CVDF SUPPORTING DATA AND CALCULATION DATABASE REV. 0

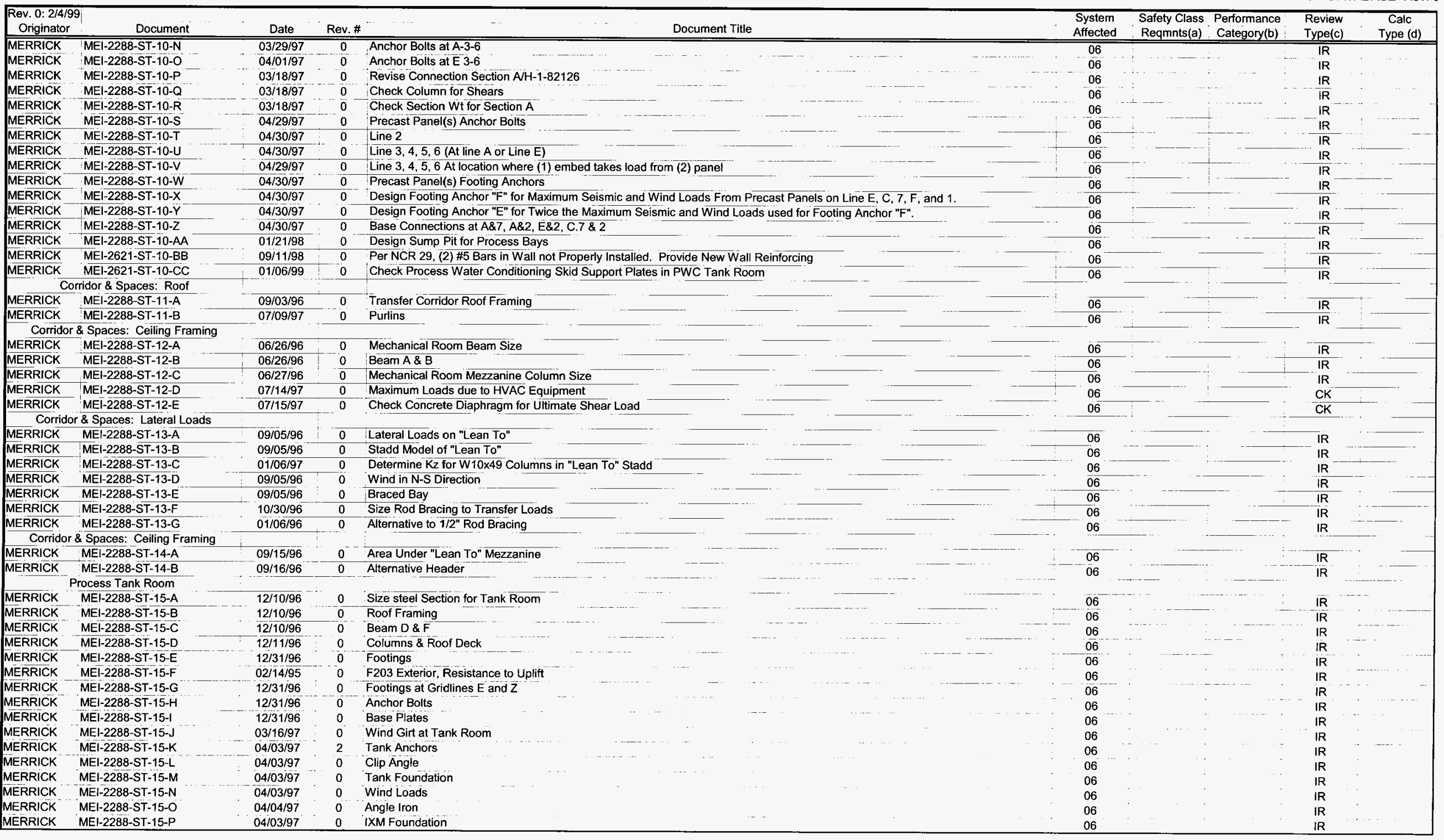




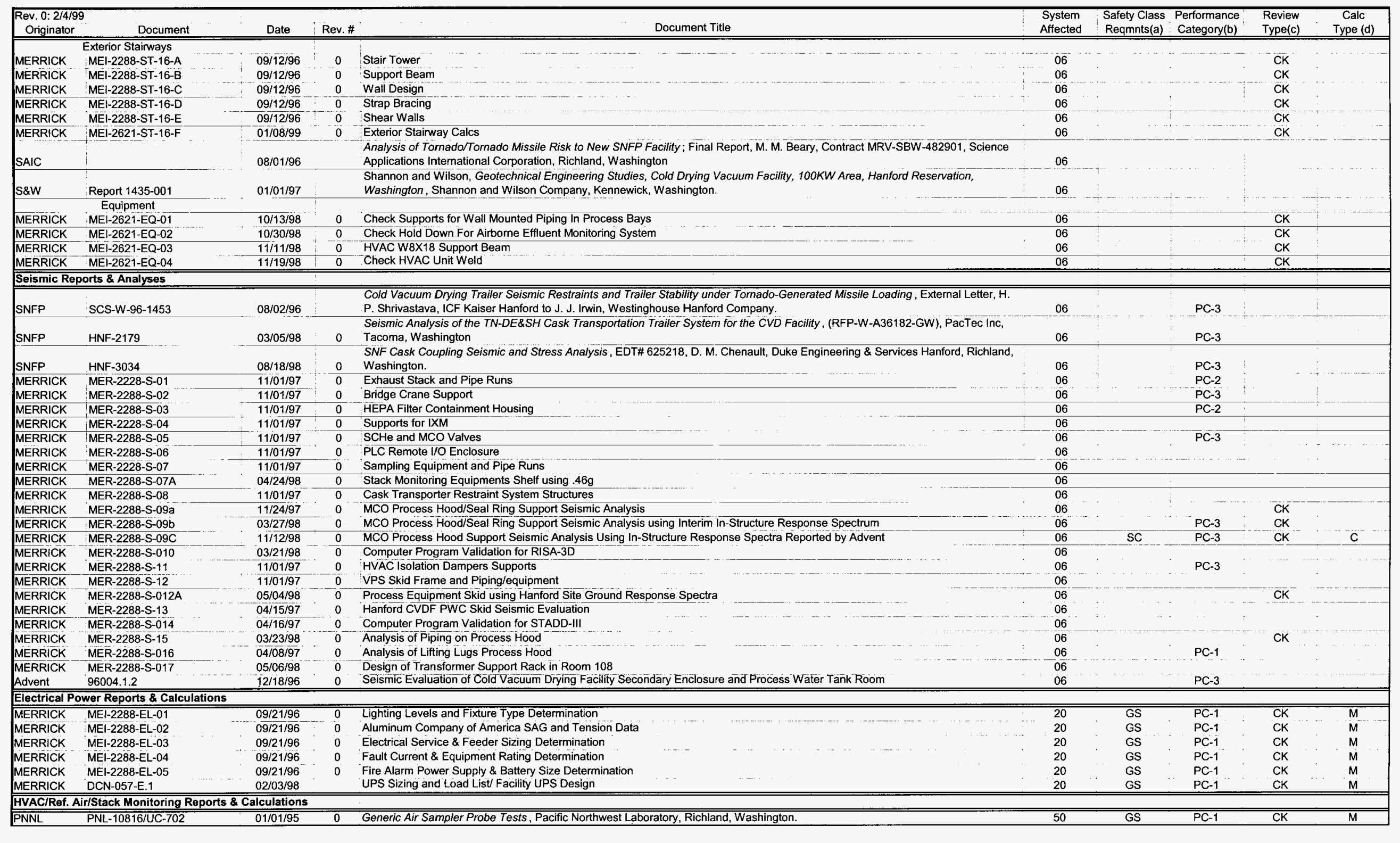


SNF-3001 CVDF SUPPORTING DATA AND CALCULATION DATABASE Rev. 0

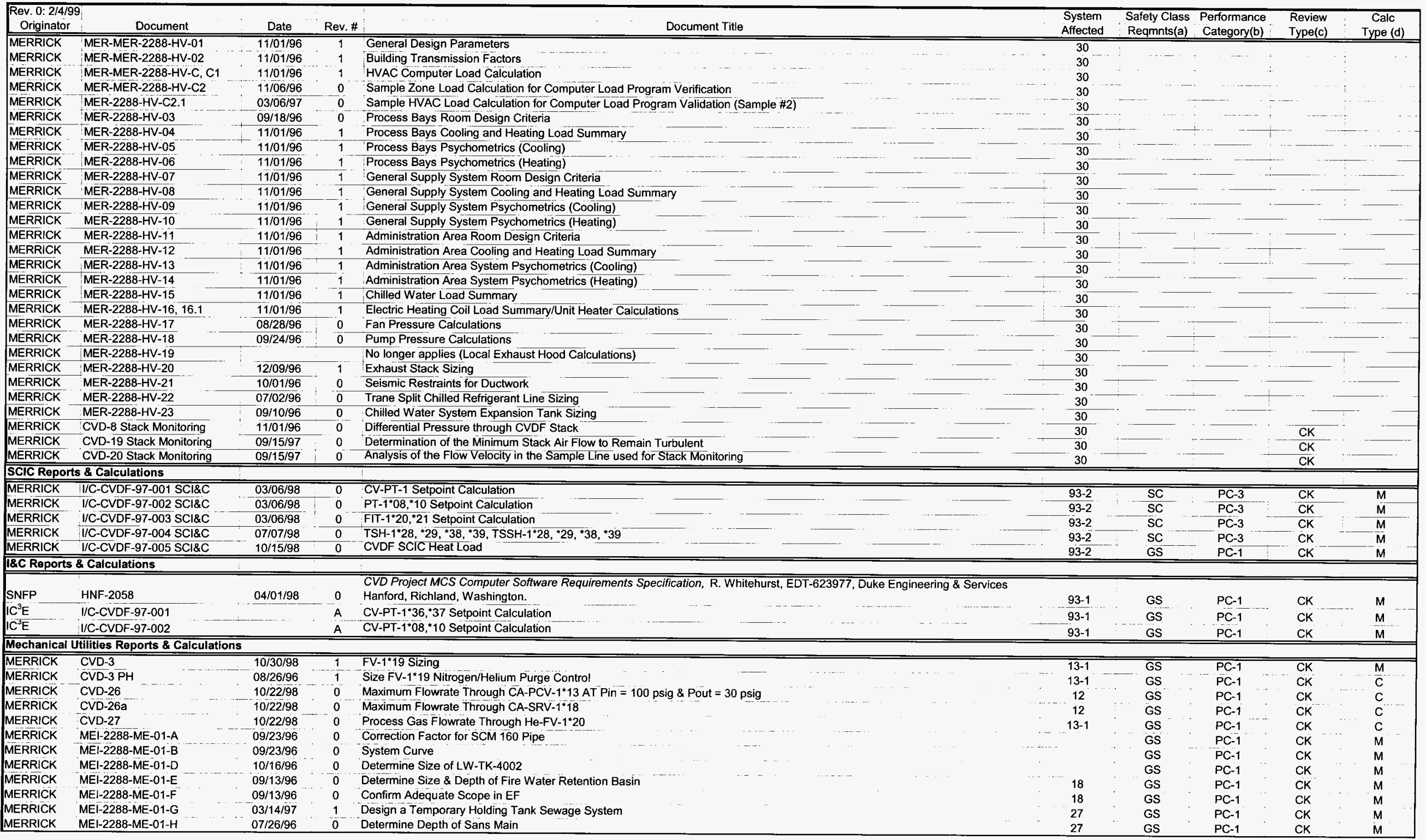


SNF-3001 CVDF SUPPORTING DATA AND CALCULATION DATABASE REV. 0

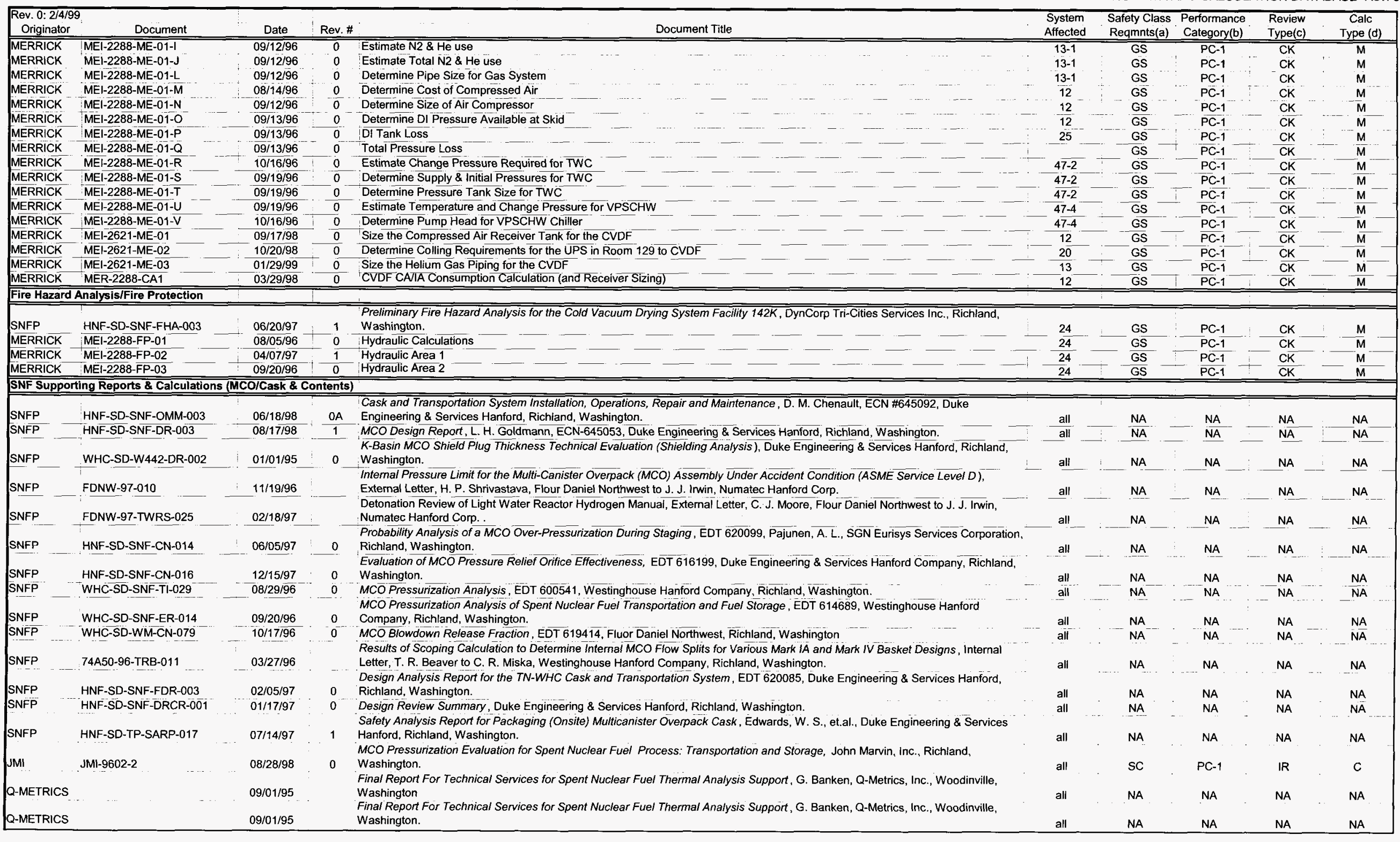




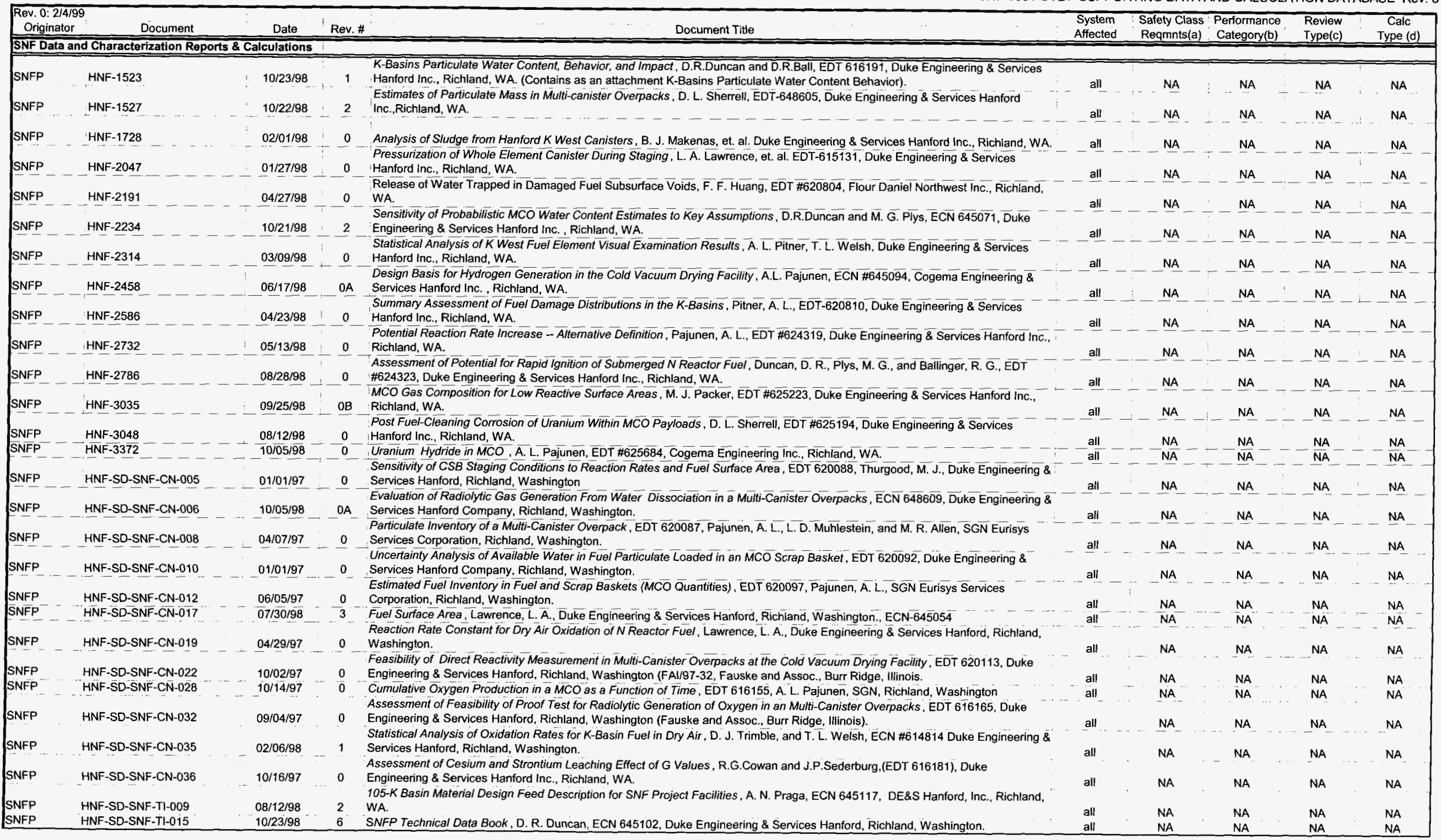




\begin{tabular}{|c|c|c|c|c|c|c|c|c|c|}
\hline \begin{tabular}{|l} 
Rev. 0: 214/99 \\
Originator \\
\end{tabular} & Document & Date & Rev. \# & Document Title & $\begin{array}{l}\text { System } \\
\text { Affected } \\
\end{array}$ & $\begin{array}{l}\text { Safety Class: } \\
\text { Requnts(a) }\end{array}$ & $\begin{array}{l}\text { Performance } \\
\text { Category(b) }\end{array}$ & $\begin{array}{l}\text { Review } \\
\text { Type(c) }\end{array}$ & $\begin{array}{c}\text { Calc } \\
\text { Type (d) }\end{array}$ \\
\hline SNFP & HNF-SD-SNF-TI-020 & $06 / 15 / 98$ & 2 & $\begin{array}{l}\text { SNFP Recommended Rate Constants for Corrosion of N-Reactor Fuel, ECN 645097, Westinghouse Hanford Company, Richland, } \\
\text { Washington. } \\
\text { MCO Internal Gas Composition and Pressure During Interim Storage, ECN-645098. Duncan, D. R. and M. G. Plys, Duke }\end{array}$ & all & NA & NA & NA & VA \\
\hline SNFP & HNF-SD-SNF-TI-040 & $10 / 23 / 98$ & 3 & $\begin{array}{l}\text { MCO Internal Gas Composition and Pressure During Interim Storage, ECN-645098, Duncan, D. R. and M. G. Plys, Duke } \\
\text { Engineering and Services Hanford, Richland, Washington. } \\
\text { in-Cell Visual Examinations of K East Fuel Elements, EDT } 620781 \text {, Duke Engineering and Services Hanford, Richland, }\end{array}$ & all & NA & NA & NA & NA \\
\hline SNFP & HNF-SD-SNF-TI-050 & $03 / 06 / 97$ & 0 & 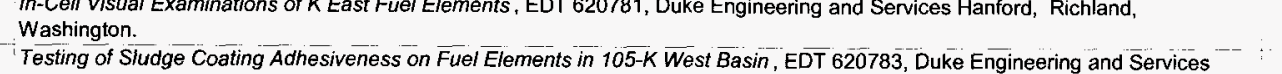 & all & NA & NA & NA & \\
\hline SNFP & HNF-SD-SNF-TRP-020 & $03 / 12 / 97$ & 0 & 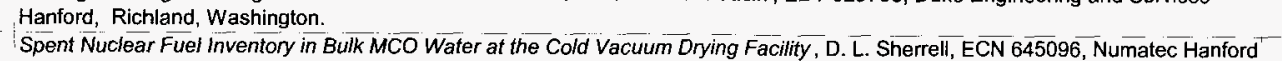 & all & - NA & NA & NA & \\
\hline $\begin{array}{l}\text { SNFP } \\
\text { SNFP- }\end{array}$ & $\begin{array}{l}\text { HNF-SD-W441-CN-001 } \\
\text { HNF-SP-1201/UC-2070 }\end{array}$ & $\begin{array}{l}06 / 11 / 98 \\
01 / 01 / 97\end{array}$ & 2 & $\begin{array}{l}\text { Corp., Richland, Washington. } \\
\text { Analysis of Sludge from K-East Basin Canisters, } \overline{D E} \& S \text { Hanford, Inc.. Richland, washington. }\end{array}$ & $\begin{array}{l}\text { all } \\
-- \text { all }\end{array}$ & $\frac{N A}{N A}-$ & $-N A$ & NA & \\
\hline $\begin{array}{l}\text { SNFP } \\
\text { SNFP }\end{array}$ & $\begin{array}{l}\text { WHC-12110-PCL93-069 } \\
\text { WHC-SD-SNF-ANAL-011 }\end{array}$ & $\frac{07 / 01 / 93}{09 / 12 / 96}$ & $\frac{0}{0}$ & $\begin{array}{l}\text { Analyses of } 105-K \text { East Basin Sludge Samples, D. B. Bechtold and M. A. Meier, Westinghouse Hanford Co., Richland, Washington } \\
\text { Comparison of Spent Fuel Databases for K-Basin. Schmittroth. F., EDT } 616570 \text {, Westinghouse Hanford CO, Richland, WA. } \\
\text { A Probabilistic Assessment of Fuel Particulate Contents of A Multi-Canister Overpack, EDT } 616141 \text {, Westinghouse Hanford }\end{array}$ & - all & $-N A$ & $-N A$ & & $\frac{N A}{N A}$ \\
\hline SNFP & WHC-SD-SNF-TI-012 & $\begin{array}{l}01 / 01 / 95 \\
02 / 01 / 95\end{array}$ & $\begin{array}{l}0 \\
0 \\
0\end{array}$ & $\begin{array}{l}\text { Summary Status of K Basins Sludge Charact } \\
\text { KE Basin Underwater Fuel Survey, A. L. Pitn }\end{array}$ & $\begin{array}{l}\text { all } \\
\text { all }\end{array}$ & $\frac{\overline{N A}}{\mathrm{NA}}$ & $\begin{array}{l}\overline{N A} \\
N A\end{array}$ & $\overline{N A}$ & $\overline{N A}$ \\
\hline SNFP & WHC-SD-SNF-TI-021 & $01 / 14 / 97$ & $1 \mathrm{~A}$ & $\begin{array}{l}\text { SNFF Detonation Phenomena of Hydrogen/Oxygen in Spent Fuel Containers, ECN } 63715 \overline{4}, \text { Westinghouse Hanford Company, } \\
\text { Richland, Washington. } \\
\text { Bounding Particulate Contents of a Mutti-Canister Overpack, Pajunen, A. L., and R. G. Cowan, ECN 638913/645044, Duke }\end{array}$ & all & NA & NA & & NA \\
\hline SNFP & WHC-SD-SNF-TI-023 & $11 / 21 / 97$ & 2 & $\begin{array}{l}\text { Engineering \& Services Hanford, Richland, Washington. } \\
\text { Multi-Canister Overpack Pressurization Monitoring and Control Methodology for the Spent Nuclear Fuei Project, EDT } 616110,\end{array}$ & & & NA & & IA. \\
\hline SNFP & WHC-SD-SNF-T1-025 & $06 / 13 / 96$ & 0 & $\begin{array}{l}\text { Westinghouse Hanford Company, Richland, Washington. } \\
\text { SNFP Surface Area Estimates for N-Reactor Fuel in the K } \bar{E} \text { Est } \overline{B a} \sin , \overline{E C N} 63 \overline{8927} \overline{7} \text {, Westinghouse Hanford Company, Richland, }\end{array}$ & all & NA & NA & NA & NA \\
\hline SNFP & WHC-SO-SNF-T1-026 & 09/30/96 & 1. & $\begin{array}{l}\text { Washington. } \\
\text { SNFP Photon Heat Deposition Calculations for Hydrogen Generation Within } \overline{M C O}, \overline{E D T} \overline{6} 16 \overline{632}, \overline{\text { Westinghouse Hanford Company. }}\end{array}$ & all & NA & NA & & NA \\
\hline SNFP & WHC-SD-SNF-TI-027 & $08 / 01 / 96$ & 0 & $\begin{array}{l}\text { Richland, Washington. } \\
\text { SNFP Gas Generation From N-Fuel in the Multi-Canister Overpacks, EDT } \overline{116} \overline{631}, \overline{\text { Westinghouse Hanford Company, Richland, }}\end{array}$ & all & NA & NA & NA & NA \\
\hline SNFP & WHC-SD-SNF-TH-028 & $08 / 01 / 96$ & 0 & Fission Products Release From Multi-Canister Overpacks, EDT $\overline{61663 \overline{3}}$ Westinghouse Hanford & all & NA & NA & NA & NA \\
\hline INFP & & -88/01/96 & 0 & & all & $\mathrm{NA}$ & NA & & $\mathrm{NA}$ \\
\hline NFP & 015 & $\begin{array}{l}07 / 25 / 95 \\
07 / 11 / 96\end{array}$ & $\begin{array}{l}1 \\
0\end{array}$ & $K \bar{E}$ & all & $\mathrm{NA}$ & $\overline{N A}$ & & $\overline{N A}$ \\
\hline SNFP & $\begin{array}{l}\text { WHC-SD-SNF-TRP-015 } \\
\text { WHC-SD-WM-ANAL-037 }\end{array}$ & $\begin{array}{l}07 / 11 / 96 \\
10 / 01 / 94\end{array}$ & $\frac{0}{0}$ & $\begin{array}{l}\text { Sludge Adherence Summary Report, ED } \\
\text { Sludge Measurement Results for } 105 \bar{K}\end{array}$ & all & $\mathrm{NA}$ & NA & & \\
\hline SAIC & WHC-SD-WUM-ANAL-UST & 10/07/94 & - & $\begin{array}{l}\text { East Basin, T. A. Meling, Westinghouse Hanford Co., Richland, Washington } \\
\text { Retrieval, Transportation and Wet-Fuei Staging, M. M. Beary, Science Applications } \\
\text { hington. } \\
\text { ontaining N Reactor Fuel, Johnson, A. B., Jr., and R. G. Clemmer, Pacific Northwest }\end{array}$ & all & NA & NA & & \\
\hline PNNL & PNNL-11341 & $01 / 01 / 96$ & 0 & $\begin{array}{l}\text { National Laboratory, Richland, Washington. } \\
\text { Examination of the Surface Coatings Removed }\end{array}$ & all & NA & $\mathrm{NA}$ & NA & \\
\hline PNNL & PNNL-11806 & 05/01/98 & 0 & $\begin{array}{l}\text { ton. } \\
\text { Furnace Testing System, Ritter, Marschman, MacFarlan and King, Pacific }\end{array}$ & all & NA & NA & NA. & NA \\
\hline PNNL & PNNL-11807 & $05 / 01 / 98$ & 0 & $\begin{array}{l}\text { Northwest National Laboratory, Richland, Washington. } \\
\text { Drying Behaviour of K-East Basin Canister Sludge, Aberfā, Marschman and Buchanan, Pacific Northwest National Laboratory, }\end{array}$ & all & NA & NA & NA & NA \\
\hline DNN & PNNL-11628 & 05/01/98 & 0 & uel Element 3128W (Run 2), Marschman, S. C., J. Abr & all & NA & NA & NA & NA \\
\hline VNL & PNNL-11818 & $01 / 01 / 98$ & 0 & $\begin{array}{l}\text { National Laboratory, Richland, Washington. } \\
\text { Oxidation Rate of K-Basin Spent Nuclear Fuel in Moist Air. Marschman, S. C., J. Al }\end{array}$ & all & NA & NA & NA & NA \\
\hline NNL & PNNL-11844 & $03 / 01 / 98$ & 0 & $\begin{array}{l}\text { Laboratory, Richland, Washington. } \\
\text { Drying Results of K-Basin Spent Nuclear Fu }\end{array}$ & all & NA & NA & IA & NA \\
\hline PNNL & PNNL-11817 & $06 / 0$ & 0 & National Laboratory, Richland, Washington. & all & NA & NA & NA & NA \\
\hline INL & & $01 / 01 / 97$ & & atory, $R$ & all & NA & NA & NA & VA \\
\hline
\end{tabular}


SNF-3001 CVDF SUPPORTING DATA AND CALCULATION DATABASE REV. 0

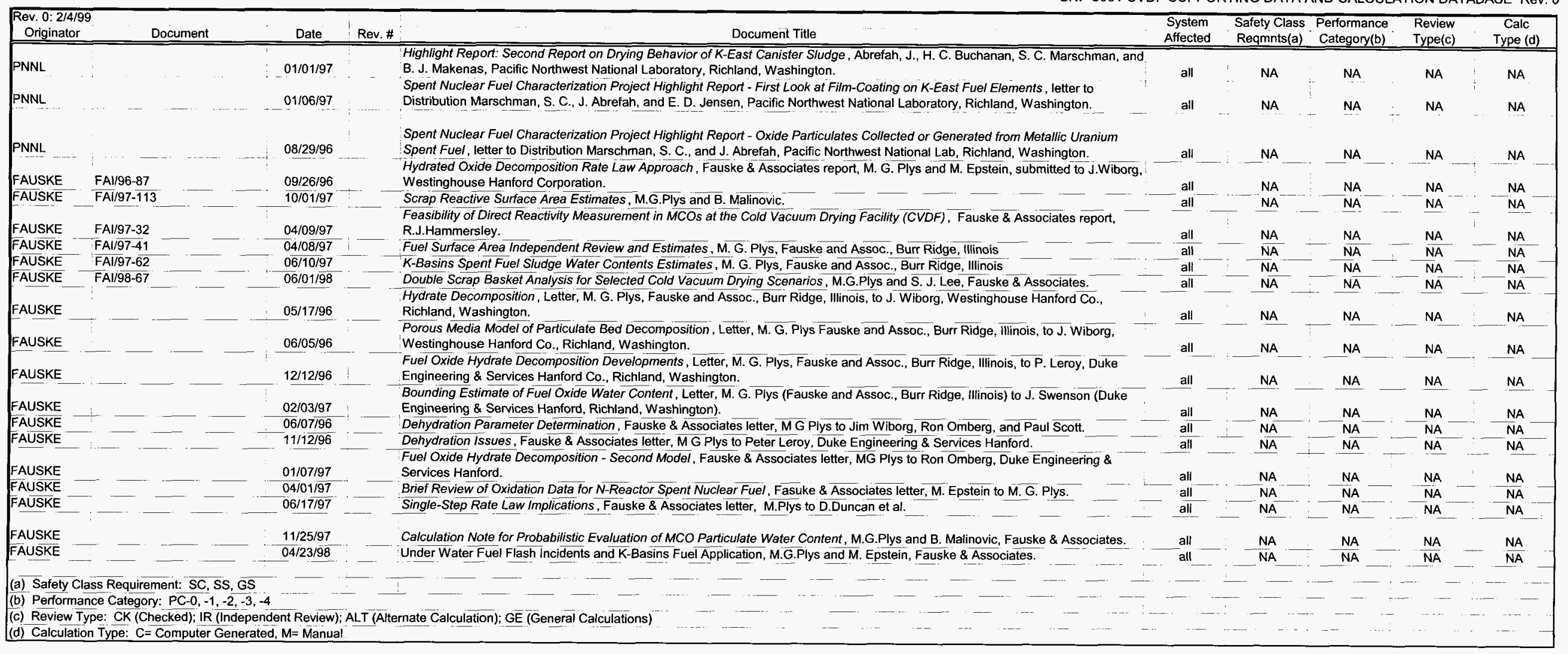




\section{DISTRIBUTION SHEET}

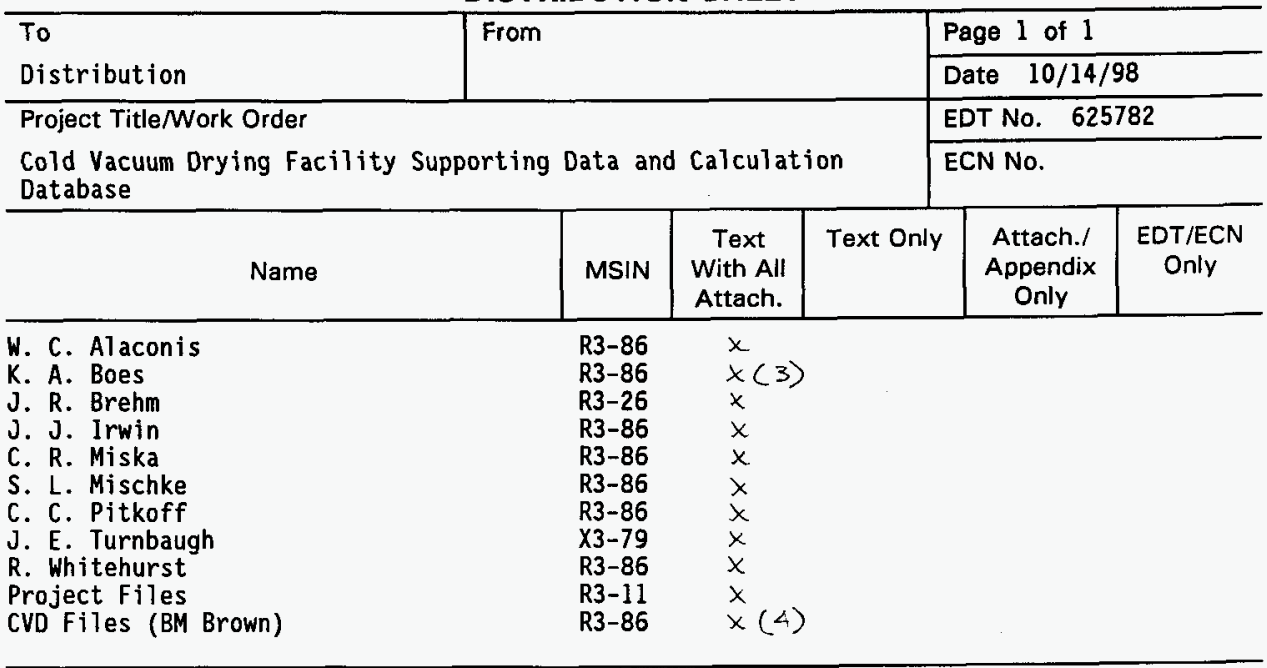

